\title{
Relación entre endoparasitismo, condición corporal y bioquímica sanguínea en monos araña (Ateles chamek) en el Centro de Rescate Taricaya, Madre de Dios, Perú
}

\author{
Relationship between endoparasitism, body condition and blood biochemistry \\ in Peruvian spider monkeys (ateles chamek) at the Taricaya Rescue Center, \\ Madre de Dios, Peru
}

\author{
Michelle Salazar ${ }^{1}$, Daniel Zárate ${ }^{1,2}$
}

\section{Resumen}

Ateles chamek (Humboldt, 1812) es una especie «En Peligro» y está incluida en el Apéndice II de la CITES, debido a la caza para comercio ilegal y deterioro de su hábitat. Por ello, se han creado centros de rescate para su protección y, en lo posible, retorno a su ambiente natural; sin embargo, el cautiverio, alimentación y manejo pueden promover el desarrollo de parasitosis. El objetivo del presente estudio fue determinar la relación entre endoparasitismo, bioquímica sanguínea y condición corporal en 20 individuos de la especie $A$. chamek del Centro de Rescate Taricaya, Madre de Dios. Se tomaron muestras de heces y sangre durante el control sanitario. La condición corporal se determinó bajo la escala basada en primates Macaca mulata. Las heces se procesaron con el método de flotación en solución de sal y azúcar; y el plasma sanguíneo fue analizado con un procesador bioquímico. En el análisis coprológico se encontró Strongyloides sp (1/20), Molineus sp (1/20), Ascaris sp (1/20), Physaloptera sp (1/20), Trichuris sp (1/20), Entamoeba sp (1/20), Giardia sp (3/20) y un nematodo ascaroideo no identificado (1/20). Se obtuvieron valores de glucosa $97.8 \pm 12.1 \mathrm{mg} / \mathrm{dl}$, proteína total $7.09 \pm 0.337 \mathrm{~g} / \mathrm{dl}$, bilirrubina total $0.12 \pm 0.0219 \mathrm{mg} / \mathrm{dl}$ y albúmina $5.47 \pm 0.416 \mathrm{~g} / \mathrm{dl}$. La condición corporal fue de $2.3 \pm 0.219$. So se encontró asociación significativa entre las variables en estudio con la parasitosis.

Palabras clave: primates, maquisapa, parásitos, bioquímica sérica, condición física

\footnotetext{
${ }^{1}$ Laboratorio de Parasitología, Área de Sanidad Animal, Departamento Académico de Nutrición, Facultad de Zootecnia, Universidad Nacional Agraria la Molina (UNALM), Lima, Perú

${ }^{2}$ E-mail: michelle.saes27@gmail.com

${ }^{3}$ E-mail: dazre@lamolina.edu.pe

Recibido: 3 de julio de 2020

Aceptado para publicación: 15 de febrero de 2021

Publicado: 24 de abril de 2021
} 
Ateles chamek (Humboldt, 1812) is an «Endangered» species and is included in Appendix II of CITES, due to poaching for illegal trade and deterioration of its habitat. For this reason, rescue centres have been created for their protection and, if possible, return to their natural environment; however, captivity, feeding and handling can promote the development of parasitosis. The aim of the present study was to determine the relationship between endoparasitism, blood biochemistry and body condition in 20 individuals of the species $A$. chamek from the Taricaya Rescue Center, Madre de Dios. Stool and blood samples were taken during the sanitary control. Body condition was determined using a score based in Macaca mulata primates. The faeces were processed with the flotation method in a salt and sugar solution; and the blood plasma was analysed with a biochemical processor. The stool analysis found Strongyloides $\mathrm{sp}(1 / 20)$, Molineus sp (1/20), Ascaris sp (1/20), Physaloptera $\mathrm{sp}(1 / 20)$, Trichuris $\mathrm{sp}(1 / 20)$, Entamoeba $\mathrm{sp}$ $(1 / 20)$, Giardia sp (3/20) and an unidentified ascaroid nematode (1/20). Glucose values were $97.8 \pm 12.1 \mathrm{mg} / \mathrm{dl}$, total protein $7.09 \pm 0.337 \mathrm{~g} / \mathrm{dl}$, total bilirubin $0.12 \pm 0.0219 \mathrm{mg} / \mathrm{dl}$ and albumin $5.47 \pm 0.416 \mathrm{~g} / \mathrm{dl}$. The body condition was $2.3 \pm 0.219$. No significant association was found between the variables under study with parasitosis.

Key words: primates, maquisapa, parasites, serum biochemistry, physical condition

\section{INTRODUCCIÓN}

El género Ateles («monos araña») pertenece a la familia Atelidae y está compuesto por siete especies, que se distribuyen a lo largo del territorio de América Central y Sudamérica (Morales-Jimenez et al., 2015). A. chamek y $A$. belzebuth son las especies presentes en el Perú. A. chamek se distribuye en los departamentos de Loreto, Huánuco, Pasco, Junín, Cusco, Ucayali, Puno y Madre de Dios, además de presentarse en Brasil y Bolivia (SERFOR, 2018).

A. chamek presenta cuerpo esbelto y miembros largos y delgados, pudiendo trepar en los estratos más altos del bosque durante la búsqueda de alimento, aunque también puede ser visto en las zonas bajas (Kellog y Goldman, 1944; Bello, 2018). La importancia de este primate radica en su rol en el ecosistema como dispersor de semillas debido a su predilección por las frutas (Van Roosmalen, 1985), además de ser especie indicadora de la calidad de los bosques. Asimismo, por su amplio rango de desplazamien- to, también es clasificada como especie emblemática y símbolo de conservación en la Amazonía peruana (WCS 2000, SERNANP 2011).

Lamentablemente, esta especie se encuentra en situación de «En Peligro», según la legislación peruana del Servicio Nacional Forestal y de Fauna Silvestre (SERFOR) (SERFOR, 2018) y por la Unión Internacional para la Conservación de la Naturaleza (UICN) (IUCN, 2016). Además, la especie está incluida en el Apéndice II de la Convención sobre el Comercio Internacional de Especies Amenazadas de Fauna y Flora Silvestre (CITES) (MINAM, 2014). La principal amenaza para este primate es la caza furtiva, con el fin de ser vendidos como mascota o «carne de monte»; y el deterioro de su hábitat, a causa de la deforestación y la invasión humana en su ecosistema, a través de la agricultura y ganadería (Soini, 1972; SERFOR, 2018). Debido a este escenario, se han creado Centros de Rescate, los cuales, mediante programas de conservación, rehabilitan y reintroducen estos animales en su hábitat. 
El Centro de Rescate Taricaya, ubicado en Madre de Dios, tiene un programa de rehabilitación y reintroducción de esta especie en la margen derecha del río Madre de Dios, área en donde se encuentra localmente casi extinto (Bello et al., 2011). Las condiciones de crianza en los centros de rescate, así como la crianza en cautiverio pueden provocar efectos negativos sobre la salud de los animales, disminuyendo la capacidad inmunológica de ellos, haciéndolos más susceptibles a infecciones parasitarias (Stoner et al. 2005; Chinchilla et al., 2007; Merck, 2007). En especies domésticas se conoce que las enfermedades parasitarias pueden alterar significativamente los valores de bioquímica sanguínea y condición corporal, tal es el caso de Fasciola hepatica, Haemonchus contortus, y Ostertagia sp, entre otros (Prache y Galtier, 1990; Yucra, 2002; Rojas, 2004; Matanoviæ et al., 2007; Guerrero et al., 2016); no obstante, esta información es escasa para el caso de especies silvestres. El objetivo del presente estudio fue determinar la relación entre endoparasitismo, bioquímica sanguínea y condición corporal en monos araña (Ateles chamek) mantenidos en cautiverio en el Centro de Rescate Taricaya, Madre de Dios.

\section{Materiales y MéTodos}

\section{Área de Estudio}

El Centro de Rescate Taricaya pertenece a la Reserva Ecológica de Taricaya, la cual se encuentra en el Departamento de Madre de Dios, distrito y provincia de Tambopata, departamento de Madre de Dios, Perú (Figura 1). Según el sistema de clasificación de Holdridge, esta zona pertenece a la categoría de bosque húmedo subtropical (bh-S). La zona corresponde a la ecorregión de Selva baja, a una altitud entre 150 y 250 msnm, y llegando a temperaturas de hasta $38^{\circ} \mathrm{C}$ (SENAMHI, 2019).
La Reserva tienen cerca de 500 ha de selva tropical y se encuentra bordeando la Reserva Nacional de Tambopata. En el Centro de Rescate se tienen animales llevados por SERFOR, provenientes de tráfico ilegal, entregas voluntarias, traslados de otras organizaciones, hallazgos y recapturas. Los animales atraviesan un protocolo estricto que consta de la recepción (evaluación de la situación de ingreso), etapa de cuarentena por 40 días como mínimo, fase de socialización donde se incorporan a grupos ya formados). Una vez que se presentan las condiciones favorables se procede a liberarlos en su hábitat, manteniendo un seguimiento constante del grupo liberado por parte de los rescatistas.

\section{Población de Estudio}

Se trabajó con la población total 20 individuos $A$. chamek presentes en el Centro de Rescate Taricaya en junio de 2018 (13 juveniles y 7 adultos, 5 machos y 15 hembras), con orígenes de recaptura y tráfico ilegal, por lo que se tenía diferencias en el tiempo de estancia de los animales. Entre ellos, 2 individuos se encontraban desde hace 4 años, 15 entre 1 y 2 años y 3 con menos de 1 año.

Todos los individuos se encontraban bajo las mismas condiciones de alojamiento, en jaulas de 10x4x3 m, acondicionadas con ramas, sogas y tableros, con una densidad de 5 a 6 individuos por jaula. El Centro de Rescate tiene un programa de desparasitación anual en el que utilizan ivermectina, praziquantel y albendazol, para el control sanitario general anual. Los individuos del estudio fueron capturados en ayuno y trasladados al tópico del Centro, en donde fueron anestesiados con una combinación de ketamina $(8 \mathrm{mg} / \mathrm{kg})$, xilacina $(0.05 \mathrm{mg} / \mathrm{kg})$ y midazolan $(0.5 \mathrm{mg} / \mathrm{kg})$ por vía intramuscular, bajo estricta supervisión veterinaria. 


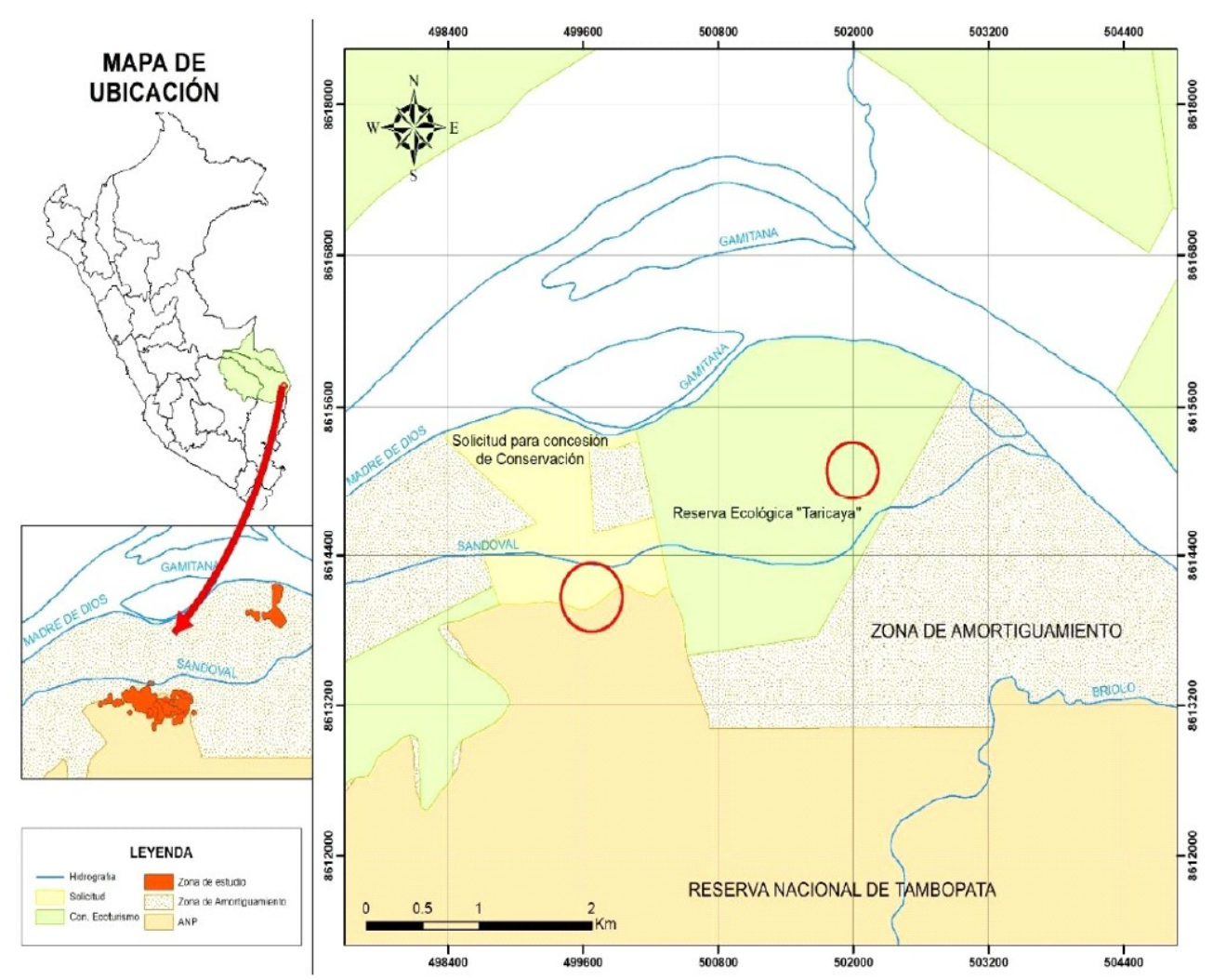

Figura 1. Mapa de la ubicación del Centro de Rescate Taricaya. Fuente: Bello (2018)

\section{Toma de Muestras}

Se tomaron muestras de heces directamente del recto mientras los animales estaban anestesiados y. en algunos casos, recolectadas de las jaulas, previa identificación (muestreo no invasivo). Las muestras de sangre fueron tomadas a través de la venopunción de la vena femoral, con una jeringa de $3 \mathrm{ml} \mathrm{y}$ aguja $\mathrm{N} .^{\circ} 21$ en tubos estériles de $1.3 \mathrm{ml}$ de capacidad conteniendo heparina. Los tubos fueron luego centrifugados a $1790 \mathrm{x} g$ durante 13 minutos y el plasma resultante fue almacenado en crioviales a $-20{ }^{\circ} \mathrm{C}$. Posteriormente, las muestras fueron trasladadas en cajas isotérmicas de poliestireno expandido al Laboratorio de Fisiología, Farmacología y Cirugía y al Laboratorio de Parasitología de la Facultad de Zootecnia de la Universidad Nacional Agraria La Molina (UNALM), Lima, para su análisis.

\section{Condición Corporal}

La condición corporal fue evaluada bajo una escala categórica basada en el primate Macaca mulata descrita por Clingerman y Summers (2005). Este procedimiento toma como referencia la distribución de grasa y músculo en el cuerpo del individuo, mediante la palpación de zonas como la pelvis, espina dorsal, masa muscular, tórax, grasa subcutánea y depósitos de grasa en la cabeza y abdomen. La asignación numérica va desde 1.0 (emaciado) al 5.0 (extremadamente obeso).

\section{Análisis de Muestras}

Las muestras de heces se analizaron a través de la técnica coprológica de sedimentación-flotación (Tello, 1988), el cual se basa en la flotación de los quistes o huevos en la 


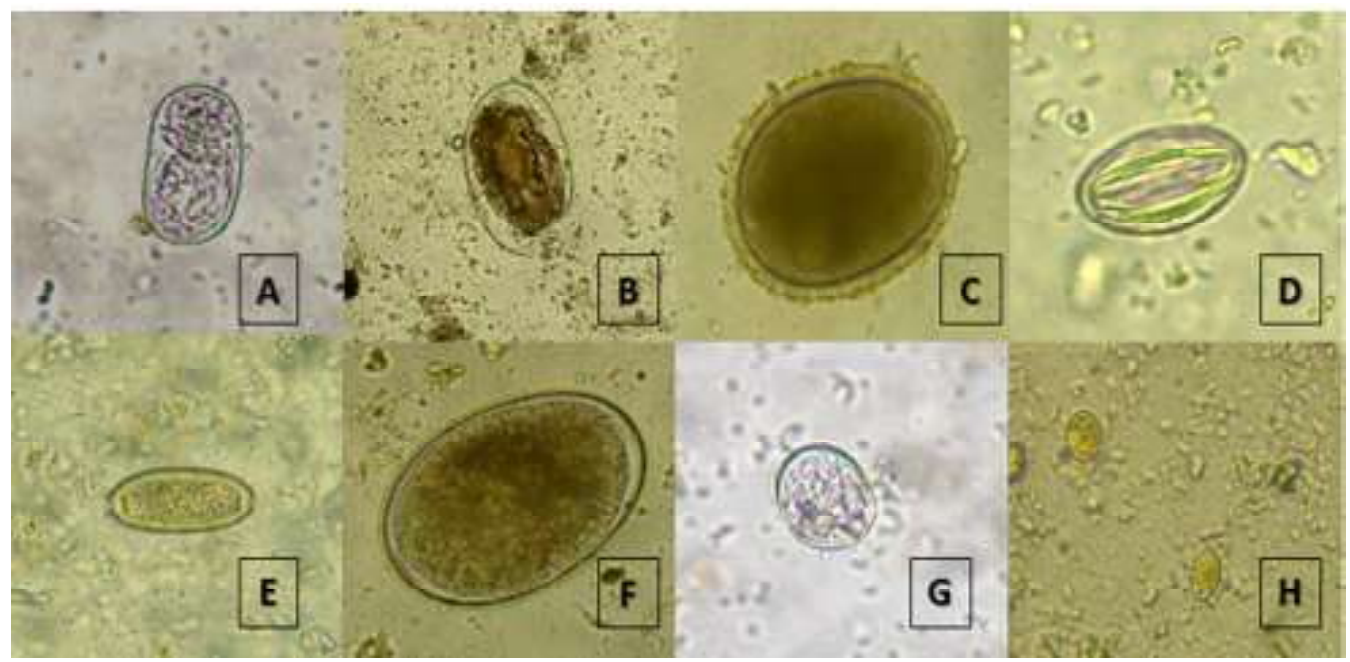

Figura 2. Huevos y quistes de parásitos gastrointestinales encontrados en monos araña (Ateles chamek) del Centro de Rescate Taricaya. A. Huevo de Strongyloides sp (40X). B. Huevo de Molineus sp (40x). C. Huevo de Ascaris sp (40X). D. Huevo de Physaloptera sp (100X). E. Huevo de Trichuris sp (40X). F. Huevo Ascaroideo no identificado (40X). G. Quiste de Giardia sp (100X). H. Quiste de Entamoeba sp $(100 \mathrm{X})$

superficie, ocasionado por la utilización de una solución altamente densa como sal o azúcar. Este método permite la detección de huevos de nematodos, cestodos y protozoarios (Beltrán et al., 2003). Las muestras de plasma sanguíneo fueron procesadas en el Analizador Bioquímico VetTest 8008 (IDEXX, USA) para la determinación de la bioquímica sanguínea (glucosa, proteína total, albúmina y bilirrubina total).

Para la identificación de los parásitos y sus huevos o quistes se utilizaron las descripciones dadas por Soulsby (1987) y Fowler (1986), mientras que para la descripción de la carga parasitaria de los individuos se empleó una escala subjetiva y no estandarizada de cruces basada en la frecuencia con que las formas parasitarias aparecen en cada campo del microscopio, la cual se resumiría en $(+)=1$ o 2 formas parasitarias en toda la muestra; $(++)=1$ a 2 formas parasitarias en la mayoría de los campos microscópicos; $(+++)=$ entre 2 a 10 formas parasitarias en cada campo observado; $(+++)=$ más de 10 formas parasitarias en cada campo observado.

\section{Análisis Estadístico}

Los resultados obtenidos fueron introducidos en el software GraphPad Prism 7.1 (GraphPad Prism Software, USA) para obtener la estadística descriptiva. En el software R (R Statical Project, Viena, Austria) se desarrolló la prueba Test de Fisher Exacto para determinar si existía asociación entre la presencia de parásitos y las variables sexo y edad, así como para evaluar la relación entre parasitosis y condición corporal. Además, se realizó la prueba t-Student para determinar la asociación entre los valores de bioquímica sanguínea (albúmina, proteína total, glucosa y bilirrubina total) y la parasitosis. 
Cuadro 1. Parásitos gastrointestinales encontrados mediante análisis coprológico en 20 monos araña negro (Ateles chamek) del Centro de Rescate Taricaya, Madre de Dios. Junio 2018

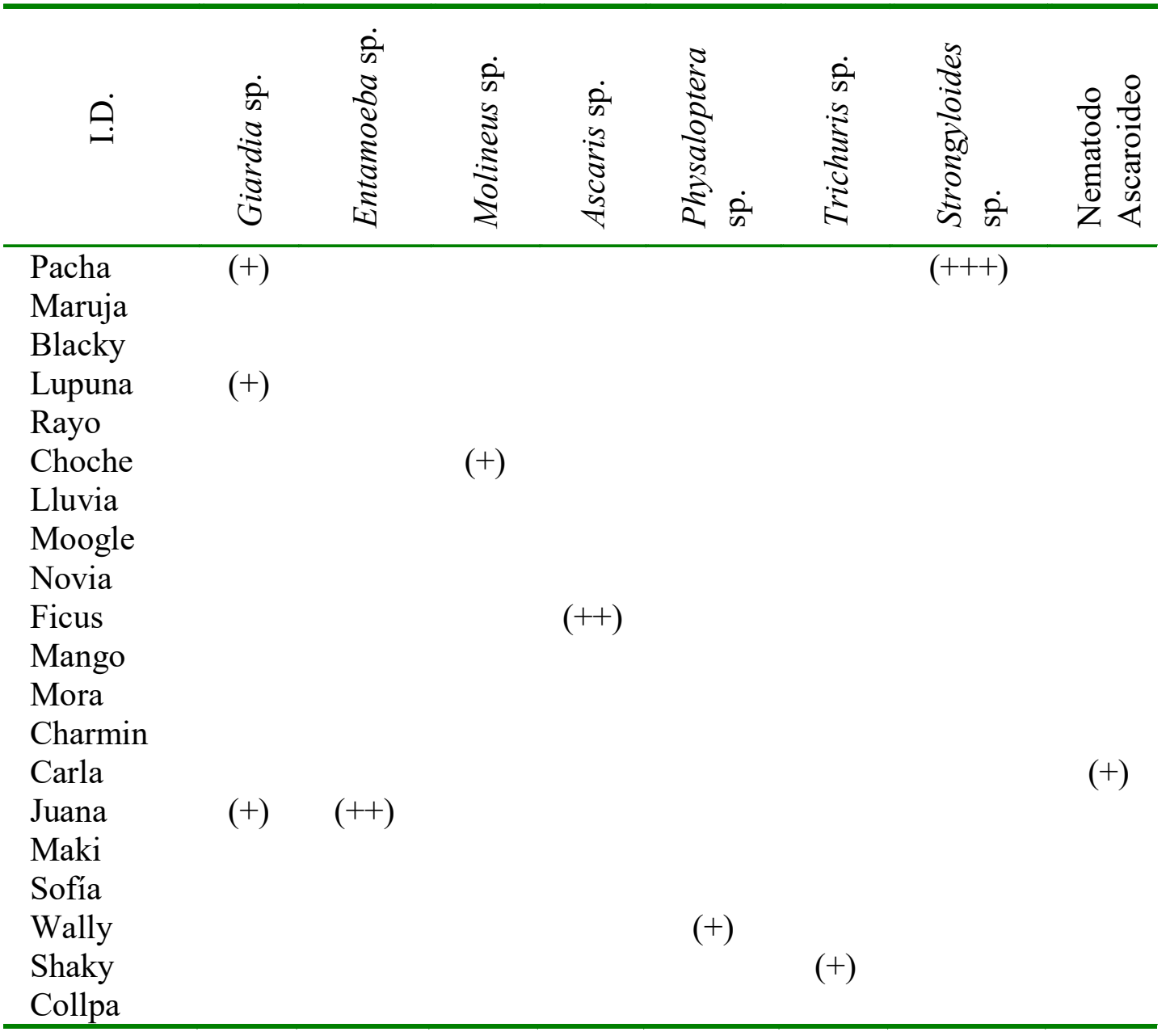

I.D.: Identificación de los individuos; Carga parasitaria expresada en cruces: (+) baja carga, $(++)$ moderada carga, (+++) alta carga

\section{Resultados}

Ocho de los 20 animales muestreados fueron positivos a la presencia de parásitos, lo que representa un $40 \%$ de la población total. Se identificaron seis nematodos gastrointestinales (Strongyloides sp, Molineus sp, Ascaris sp, Physaloptera sp, Trichuris sp y un Ascaroideo no identificado debido a la descorticación del huevo, y dos protozoarios (Giardia sp y Entamoeba sp) (Figura 2; Cuadro 1). Giardia sp fue el parásito más frecuente y el nematodo Strongyloides sp el que tuvo la mayor carga parasitaria por individuo.

El análisis estadístico determinó que no existía relación significativa $(\mathrm{p}>0.05)$ entre la infección parasitaria y las variables de sexo 
y edad. Tampoco se encontró asociación entre la infección parasitaria y la condición corporal. Los valores promedio ( \pm IC al 95\%) de la bioquímica sanguínea fueron de glucosa $97.8 \pm 12.1 \mathrm{mg} / \mathrm{dl}$, proteína total $7.09 \pm 0.337$ $\mathrm{g} / \mathrm{dl}$, bilirrubina total $0.12 \pm 0.0219 \mathrm{mg} / \mathrm{dl}$ y albúmina $5.47 \pm 0.416 \mathrm{~g} / \mathrm{dl}$. La condición corporal promedio fue $2.3 \pm 0.219$. No se encontró asociación significativa entre las variables en estudio.

\section{Discusión}

La diversidad de parásitos encontrados en el presente estudio fue mayor a la encontrada por investigaciones realizadas en la misma especie de primate, incluyendo aquellas en condiciones de silvestría. Por ejemplo, Carrasco et al. (2008) evaluaron tres grupos sociales de $A$. chamek en silvestría en Madre de Dios encontrando cinco especies de helmintos (Strongyloides sp, Trichuris sp, Trypanoxyuris sp, nematodo Ancylostomidae y trematodo Dricoeliidae); Phillips et al. (2004) encontraron dos nematodos en la Reserva Nacional de Tambopata (Strongyloides sp y Trichuris sp) y tres protozoarios (Chilomastix, Blastocystis e Iodamoeba) en individuos $A$. chamek en silvestría; en tanto que Guerrero et al. (2012) identificaron tres nematodos (Trichostrongylidae, Strongyloides cebus y un Oxyuroideo) y 1 cestodo (Paratriotaenia oedipomidatis) en monos araña bajo cautiverio. En estos estudios se observa una variada fauna parasitaria. El presente estudio representa uno de los primeros reportes para las especies de nematodos Molineus sp y Physaloptera sp en A. chamek. Molineus sp solo ha sido hallado en primates neotropicales como Saimiri sciureus, Saguinus mystax, Saguinus nigricollis y Cebus spp (Sarmiento et al., 1999), mientras que Physaloptera sp ha sido registrado en monos choro (Lagothrix poeppiggi) (Fernández, 2011).

Si bien se registró una diversa fauna parasitaria, las cargas encontradas fueron moderadas a bajas, debido a que algunos animales habían sido recapturados y recibido tratamiento antiparasitario. La presencia de protozoarios parásitos podría indicar un contacto cercano entre estos animales y los cuidadores (Appelbee et al., 2005; Carrasco et al., 2008; Chinchilla et al., 2007), como fue demostrado por Fernández (2011), quien encontró Giardia sp en muestras de un mono choro y en humanos, por lo que representan un potencial problema zoonótico para el Centro de Rescate.

Estudios previos indican que las hembras son más susceptibles a ser infectadas por parásitos, debido a que su sistema inmunológico puede verse afectado por el estrés causado durante su migración a otros grupos al llegar a la edad juvenil, la maternidad y las agresiones físicas que sufren por parte de los machos (Campbell, 2003; Castañeda et al., 2010). Asimismo, se reportan mayores cargas parasitarias en individuos de mayor edad (Castañeda et al., 2010; Benavides et al., 2012). No obstante, en el presente estudio no encontró evidencia estadística que demuestre que las hembras sean más afectadas que los machos o que la edad influya en la parasitosis del individuo, posiblemente debido a las condiciones de manejo y de cautiverio de los animales en el presente trabajo.

En cuanto a la relación de parasitosis y condición corporal, se podría esperar que la infección parasitaria provoque que los individuos disminuyan su condición física como efecto negativo de la presencia de parásitos. En el estudio de Benavides et al. (2012) en primates del viejo mundo, se encontró una correlación negativa entre condición la corporal y fauna parasitaria, aunque Agostini et al. (2017) no lograron mejorar la condición (masa) corporal al aplicar antiparasitarios en capuchinos negros (Sapajus nigritus). De manera semejante, en el presente estudio no se halló asociación entre condición corporal y parasitosis, posiblemente debido a las cargas parasitarias bajas que presentaban los monos araña. 
Los valores bioquímicos encontrados coinciden con anteriores estudios realizados en esta especie de primate (Karesh et al., 1998; Dávila et al., 2014; Ríos, 2015). Si bien no hay información reportada acerca de la relación valores bioquímicos-parasitosis en primates, investigaciones realizadas en humanos han registrado efecto de las infecciones por parásitos sobre algunos parámetros bioquímicos. Así, en un estudio en niños infectados con Giardia lamblia se observó una disminución en la glucosa sanguínea, atribuyendo este efecto a la glucosa consumida por los trofozoitos durante su crecimiento (Yahya et al., 2007). De igual forma, Abdul-Hussien (2005) en humanos con giardiasis, evidenció la disminución de los niveles de albúmina, a causa del daño a nivel de lumen intestinal y que desencadenó en sucesivas diarreas agudas y crónicas, lo que terminó afectando el nivel de proteína total sanguínea. El presente estudio no evidenció asociación estadística entre las variables de parámetros bioquímicos y parasitosis ni entre la condición corporal y la parasitosis, lo cual podría atribuirse a la baja carga parasitaria encontrada.

\section{Conclusiones}

- No se encontró relación estadística entre la parasitosis y la condición corporal de monos araña (Ateles chamek).

- No se encontró relación estadística entre los valores bioquímicos obtenidos (glucosa, proteína total, bilirrubina total y albúmina) y la parasitosis.

- La fauna parasitaria encontrada fue variada, incluyendo seis especies de nematodos y dos protozoarios, incluyendo dos nuevos hallazgos de nematodos para A. chamek (Physaloptera sp y Molineus sp).

\section{Agradecimientos}

Los autores agradecen al apoyo del Centro de Rescate Taricaya, en especial al Regente Raúl Bello, por las sugerencias, apreciaciones y facilidades brindadas en la realización de este trabajo. A la Oficina de Extensión Universitaria de la UNALM, que bajo gestión del profesor Segundo Gamarra subvencionaron los pasajes hacia la Reserva y la utilización del Analizador Bioquímico VetTest 8008. Al grupo de estudiantes de la Universidad de Tufts, USA, por la ayuda con la recolección de muestras, y a A. Gonzalo Suárez por la ayuda con el análisis estadístico.

\section{Literatura Citada}

1. Abdul-Hussien AJK. 2005. Effect of giardia on some biochemical variables in human blood serum. AJPS 2: 29-35.

2. Agostini I, Vanderhoeven E, Di Bitetti MS, Beldomenico PM. 2017. Experimental testing of reciprocal effects of nutrition and parasitism in wild black capuchin monkeys. Nature Sci Reports 7: 12778.

3. Appelbee AJ, Thompson RA, Olson $M E$. 2005. Giardia and Cryptosporidium in mammalian wildlife-current status and future needs. Trends in parasitology,21(8), 370-376.

4. Bello R. 2018. Comportamiento de monos arañas (Ateles chamek) reintroducidos en el sureste de la Amazonía peruana. Tesis de Maestría. Lima, Perú: Univ. Nacional Agraria La Molina. 88 p.

5. Bello R, Timson S, Rosemberg F, Escate W. 2011. Rehabilitación y Reintroducción del mono araña, Ateles chamek (Humboldt, 1812), en el sureste de la Amazonia Peruana. Puerto Maldonado, Perú: Centro de Rescate Taricaya. $19 \mathrm{p}$.

6. Beltrán M, Tello R, Náquira C. 2003. Manual de procedimientos de laboratorio para el diagnóstico de los parásitos intestinales del hombre. Serie de Normas Técnicas $\mathrm{N}^{\circ}$ 37. Lima: Instituto Nacional de Salud. 90 p.

7. Benavides JA, Huchard E, Pettorelli $N$, King AJ, Brown ME, Archer CE, et al. 2012. From parasite encounter to infection: multiple scale drivers of 
parasite richness in a wild social primate population. Am J Phys Anthropol 147: 52-63. doi: 10.1002/ajpa.21627

8. Campbell CJ. 2003. Female-directed aggression in free-ranging Ateles geoffroyi. Int J Primatol 24: 223-237.

9. Carrasco F, Tantaleán M, Gibson KN, Williams M. 2008. Prevalencia de helmintos intestinales de una población de monos maquisapas silvestres Ateles belzebuth chamek en el Parque Nacional de Manu, Perú. Neotrop Helminthol2: 19-26.

10. Castañeda FE, Rubiano JO, Cruz LJ, Rodriguez LC. 2010. Prevalencia de helmintos intestinales en primates neotropicales cautivos alojados en la ciudad de Ibagué. Rev Colomb Cienc Anim 3: 34-40.

11. Chinchilla M, Guerrero OM, Gutiérrez-Espeleta GA, Sánchez R, Valerio Campos I. 2007. Parásitos en monos carablanca Cebus capucinus (Primates: Cebidae) de Costa Rica.Parasitol Latinoam 62: 170-175.

12. Clingerman K.J, Summers L. 2005. Development of a body condition scoring system for nonhuman primates using Macaca mulatta as a model. Lab Animal 34: 31-36. doi: 10.1038/laban0505-31

13. Dávila R, Navarrete M, Li O, Lira B, Gomez L, Hoyos L. 2014. Estudio hematológico, bioquímico, parasitológico, electrocardiográfico y de presión arterial del mono maquisapa (Ateles chamek) criado en semicautiverio en Tarangue, Moyobamba. En: XXIV Congreso Panamericano Ciencias Veterinarias. Cuba.

14. Fernández D. 2011. Parasitismo gastrointestinal del mono choro (Lagothrix poeppigii) y de los pobladores amazónicos locales del río Yavarí Mirín, Loreto. Tesis de Médico Veterinario. Lima, Perú: Univ. Nacional Mayor de San Marcos.

15. Fowler E. 1986. Zoo and wild animal medicine. $2^{\circ}$ ed. Elsevier.

16. Guerrero F, Serrano-Martínez $E$, Tantaleán M, Quispe M, Casas G. 2012. Identificación de parásitos gastrointestinales en primates no huma- nos del zoológico parque natural de Pucallpa, Perú. Rev Inv Vet Perú 23: 469-478.

17. Guerrero L, Rossini M, Bethencourt $A$, Colmenares $O$, Rueda de Arvelo $E$, Ríos de Álvarez L. 2016. Efecto de la suplementación con semilla de Canavalia ensiformis sobre parámetros sanguíneos de ovinos tropicales con infecciones parasitarias castrointestinales. Rev Fac Cienc Vet 57: 101-113.

18. IUCN. 2016. IUCN Red List of threatened species. Wallace RB, Mittermeier RA, Cornejo F, Boubli J. 2015. Ateles chamek. Disponible en http://www.iucnredlist.org

19. Karesh WB, Wallace RB, Painter RLE, Rumiz D, Braselton WE, Dierenfeld ES, Puche H. 1998. Immobilization and hvalth assessment of free ranging black spider monkeys (Ateles paniscus chamek). Am J Primatol 44: 107-123. doi: 10.1002/(SICI)10982345 ( 1998$) 44: 2<107$ : : A ID AJP2>3.0.CO;2-\#

20. Kellog R, Goldman EA. 1944. Review of the spider monkeys. Proc United States National Museum 96: 1-45. doi: 10.5479/si.00963801.96-3186.1

21. Matanoviae K, Severin K, Martinkoviae F, Šimpraga M, Janicki Z, Barišice J. 2007. Hematological and biochemical changes in organically farmed sheep naturally infected with Fasciola hepatica. Parasitol Res 101: 1657-1661. doi: 10.1007/s00436-007-0709-2

22. Merck. 2007. Manual Merck de Veterinaria. [Internet]. Disponible en: https:// www.merckvetmanual.com/

23. [MINSAM] Ministerio del Ambiente. 2014. Especies de fauna silvestre peruana en los apéndices de la CITES. Actualización del listado de Especies de Fauna Silvestre Peruana en los Apéndices de la CITES luego de la Decimosexta Reunión de la Conferencia de las Partes (CoP 16). Versión 1.1.

24. Morales-Jimenez, AL, Disotell T, Di Fiore A. 2015. Revisiting the phylogenetic relationships, biogeography, 
and taxonomy of spider monkeys (genus Ateles) in light of new molecular data. Mol Phylogenet Evol 82: 467-483. doi: 10.1016/j.ympev.2014.09.019

25. Phillips KA, Haas ME, Grafton BW, Yrivarren M. 2004. Survey of the gastrointestinal parasites of the primate community at Tambopata National Reserve, Peru. J Zoology 264: 149-151. doi: $10.1017 / \mathrm{S} 0952836904005680$

26. Prache S, Galtier P. 1990. Changes in blood bilirubin and plasma activity of gamma-glutamyl transferase in lambs experimentally infested with Fasciola hepatica. Reprod Nutrit Dev (Suppl 2): 233s-234s.

27. Ríos F. 2015. Valores hematológicos y bioquímicos del mono araña negro (Ateles chamek) en cautiverio en el departamento de Madre de Dios. Tesis de Médico Veterinario. Lima, Perú: Univ. Alas Peruanas. $64 \mathrm{p}$.

28. Rojas C. 2004. Nosoparasitosis de los rumiantes domésticos peruanos. $2^{\circ} \mathrm{ed}$. Lima, Perú: Martegraf. $42 \mathrm{p}$.

29. Sarmiento L, Tantaleán M, Huiza A. 1999. Nematodos parásitos del hombre y de los animales en el Perú. Rev Per Parasitol 14: 9-65.

30. [SERFOR] Servicio Nacional Forestal y de Fauna Silvestre. 2018. Libro rojo de la fauna silvestre amenazada del Perú. SERFOR. Lima. Perú. 558 p.

31. [SERNANP] Servicio Nacional de Áreas Naturales Protegidas. 2011. Diagnóstico del proceso de elaboración del plan maestro 2011-2016 de la Reserva Nacional Tambopata. [Internet]. Disponible en: http://old.sernanp.gob.pe/ sernanp/archivos/biblioteca/planesmaestros_2012/PM\%20RNTAMB\%202011-2016.pdf

32. Soini P. 1972. The capture and commerce of live monkeys in the Amazo- nian region of Peru. nternat Zoo Yearbook 12: 26-36. doi: 10.1111/j.17481090.1972.tb02260.x

33. Soulsby E. 1987. Parasitología y enfermedades parasitarias en los animales domésticos. $7^{\circ}$ ed., México DF. Interamericana. $823 \mathrm{p}$.

34. Stoner KE, González-Di Pierro AM, Maldonado-López S. 2005. Infecciones de parásitos intestinales de primates: implicaciones para la conservación.- Universidad y Ciencia II: 61-72.

35. Tello R. 1988. Empleo de una nueva técnica parasitológica rápida de sedimentación espontánea en el diagnóstico de protozoarios y helmintos. En: V Jornadas Científicas. Lima.

36. Van Roosmalen MG. 1985. Habitat preferences, diet, feeding strategy and social organization of the black spider monkey [Ateles paniscus paniscus Linnaeus 1758] in Surinam. Acta Amaz 15: 7-238. doi: 10.1590/180943921985155238

37. [WCS] Wildlife Conservation Society. 2000. Programa de los paisajes vivientes, Área de conservación del paisaje de los Andes del Noroeste boliviano - Informe Anual de Actividades, Bolivia. [Internet]. Disponible en: https:// bolivia.wcs.org/Portals/14/Informes Anuales/In forme Anua lWCS2000.pdf

38. Yucra V. 2002. Carga parasitaria gastrointestinal, lesiones anatomohistopatológicas, respuesta celular y patrón de respuesta humoral en alpacas de una comunidad campesina-Puno. Tesis de Magíster. Lima, Perú: Univ. Nacional Mayor de San Marcos. 82 p.

39. Yahya GS, Esraa AM, Majida NI 2007. Effect of Giardia lamblia on some biochemical changes of the human. Tikrit J Pure Sci 12: 29-32. 